\title{
Rapid Detection of Mycobacterium tuberculosis in PLHIV and Its Correlation with CD4 Count
}

\author{
Mousumi Kilikdar*, Dilip Gedam, Ashwini Pisey and Rajesh P. Karyakarte
}

Department of Microbiology, Government Medical College, Akola, Maharashtra, India

*Corresponding author

\section{A B S T R A C T}

\section{Keywords}

Mycobacterium tuberculosis, Ziehl

Nielsen stain and

GeneXpert

\section{Article Info}

Accepted:

04 June 2019

Available Online:

10 July 2019
HIV and TB co-infection is an alarming situation in the health care system. India has the third largest number of people living with HIV (PLHIV) in the world. This co-infection leads to difficulty in both diagnosis and treatment of TB resulting in increased mortality, morbidity, treatment failure and relapse. The present study was conducted to determine the prevalence of TB in PLHIV and also to determine its co-relation with the CD4 counts of the affected individuals. This is a prospective study conducted over a period of one year commencing from April 2017 to March 2018. Sputum and blood samples were collected from 288 HIV positive individuals who were clinically suspected to have TB as coinfection and samples were sent to Department of Microbiology, GMC Akola. Sputum analysis was done by Ziehl Nielsen stain and GeneXpert. CD4 counting of blood sample was done by Flow Cytometer. Among 288 individuals participated in the study, 147 individuals were associated with HIV TB co-infection. 115 patients out of $288(39.93 \%)$ were positive by CBNAAT and $32(11.11 \%)$ were positive by sputum microscopy for acid fast bacilli. Out of these 115 CBNAAT positive patients, $23(20 \%)$ showed rifampicin resistance. About 96 (65.30\%) HIV and TB co infected individuals had a CD4 counts <200 cells/ $\mu$ l followed by $35(23.80 \%)$ individuals with CD4 counts ranging between 200-300 cells/ $\mu \mathrm{l}$ and in only $2(1.36 \%)$ cases CD4 counts were more than 500 cells/ $\mu 1$. The present study emphasizes that co-infection with TB has been a major concern in HIV/AIDS patients. TB is the the most common opportunistic infection in HIV positive patients even at higher CD4 counts and most of the time remains smear negative. Hence early diagnosis and optimum management is necessary to reduce mortality and morbidity.

\section{Introduction}

HIV and TB co-infection is an alarming situation in the health care system. Mycobacterium tuberculosis in coordination with HIV enhances the descent of immunological functions and pathogenicity of one another leading to death if left untreated ${ }^{1}$.
The mechanism involved in the progression of HIV TB co-infection on host's immune system has not described completely. Inborn error of immunity and genetic polymorphism are few factors affecting this progression ${ }^{1}$. India has the world's highest burden of TB and third largest number of people living with HIV (PLHIV) in the world. It is estimated that 
$60-70 \%$ of HIV positive person will develop tuberculosis in their lifetime ${ }^{2}$. Globally about 476774 cases of TB were detected in people living with human immunodeficiency virus infection (PLHIV) by the end of $2016^{3}$. The incidence of HIV TB co-infection is 1,63000 and the mortality due to this co-infection was about 35 thousands in South East Asia as per the estimation of WHO done in $2016^{3}$.

About one third of HIV-TB cases goes underdiagnosed as there is scanty sputum production, lack of caseous necrosis leading to decreased number of bacilli in sputum and thus reducing sensitivity of smear microscopy ${ }^{4}$. Culture result for TB is available only after 2-8 weeks. Xpert MTB/RIF, holds a good promise by detecting $99 \%$ of smear-positive patients and $>80 \%$ of patients with smear negative disease ${ }^{5}$.

The pattern of clinical presentation of TB depends on the host immune status. The CD4 $\mathrm{T}$ cell count is one of the best indicators of immunological competence of the patient with HIV infection. Thus determination of CD4 cell counts provides a powerful tool for determining the prognosis and identification, assessment and induction of prophylactic therapies for various opportunistic infections in high risk individual. The present study was conducted to determine the prevalence of TB in PLHIV and also to determine its co-relation with the CD4 counts of the affected individuals.

\section{Materials and Methods}

The prospective hospital based study was conducted in the department of Microbiology, Government Medical College Akola, Maharashtra, India from April 2017 to March 2018 in association with antiretroviral therapy (ART) centre, Integrated Counselling \& Testing Centre of our hospital. HIV positive patients above the age of 18 years having manifestations of TB (cough for less than or up to 2 weeks) were included in the study. A total of $750 \mathrm{HIV}$ positive individuals attended the ART centre for regular follow up out of which 288 individuals were clinically suspected to have TB as co-infection.

\section{Immune status assessment}

About $3 \mathrm{ml}$ of blood was collected from each patient using aseptic precaution in ART centre and sent to CD4 lab. CD4 counting of blood sample was done by Flow Cytometer (PartecCyFlow Counter) as per manufacturer's instructions.

\section{Sputum analysis}

Two samples of at least $1 \mathrm{ml}$ sputum were obtained from each patient, in 2 sterile containers and sent to Microbiology department. One was used for Ziehl Nielsen stain. Another one was diluted with three times the reagent, incubated at room temperature and loaded into Xpert MTB/RIF cartridge for automated analysis. Results were obtained in 2 hours. Detection of Mycobacteria and rifampicin resistance was carried out in the same setting.

\section{Results and Discussion}

The most commonly affected age group was 21-40 years. Males were most commonly affected $(88,66 \%)$. The age and gender wise distribution were depicted in the table 1. Among 288 individuals participated in the study, 147 individuals were associated with HIV TB co-infection (Figure 1).

115 patients out of $288 \quad(39.93 \%)$ were positive by CBNAAT and $32(11.11 \%)$ were positive by sputum microscopy for acid fast bacilli. Out of these 115 CBNAAT positive patients, 23 (20\%) showed rifampicin resistance (Figure 2). 
About 96 (65.30\%) HIV and TB co infected individuals had a CD4 counts $<200$ cells $/ \mu 1$ followed by $35(23.80 \%)$ individuals with CD4 counts ranging between 200-300 cells/ $\mu 1$ and in only $2(1.36 \%)$ cases CD4 counts were more than 500 cells $/ \mu 1$. The association of CD4 counts with the occurrence of Pulmonary Tuberculosis is shown in table 2.
In our study, male preponderance was seen which is in concordance with other studies like Rao et al., ${ }^{6}$, Sharma et al., ${ }^{7}$.This could be due to migration for employment and their high risk behaviour. The most commonly affected age group was 21-40 years which is similar to the findings of Nara et al., ${ }^{8}$ and Siddeshwari et al., ${ }^{9}$.

Table.1 Age distribution of patients

\begin{tabular}{|c|c|c|c|}
\hline \multirow[t]{2}{*}{ Age group } & \multicolumn{2}{|c|}{ Gender } & \multirow[t]{2}{*}{ Percentage (n\%) } \\
\hline & Male & Female & \\
\hline $18-20$ & 8 & 3 & $7.48 \%$ \\
\hline $21-40$ & 45 & 33 & $53 \%$ \\
\hline $41-60$ & 25 & 21 & $31.29 \%$ \\
\hline 61 and above & 10 & 2 & $8.16 \%$ \\
\hline Total & 88 & 59 & $100 \%$ \\
\hline
\end{tabular}

Table.2 Association of CD4 counts with the occurrence of Pulmonary Tuberculosis

\begin{tabular}{|l|l|}
\hline \multicolumn{1}{|c|}{ Counts: Cells/ $\boldsymbol{\mu l}$} & \multicolumn{1}{c|}{ HIV with Pulmonary Tuberculosis(n=147) } \\
\hline$<\mathbf{2 0 0}$ & $96(65.30 \%)$ \\
\hline $\mathbf{2 0 0 - 3 0 0}$ & $35(23.80 \%)$ \\
\hline $\mathbf{3 0 1 - 5 0 0}$ & $14(9.52 \%)$ \\
\hline$>\mathbf{5 0 0}$ & $2(1.36 \%)$ \\
\hline Total & $\mathbf{1 4 7}(\mathbf{5 1 . 0 4 \% )}$ \\
\hline
\end{tabular}

Fig.1 HIV (288), TB (147)

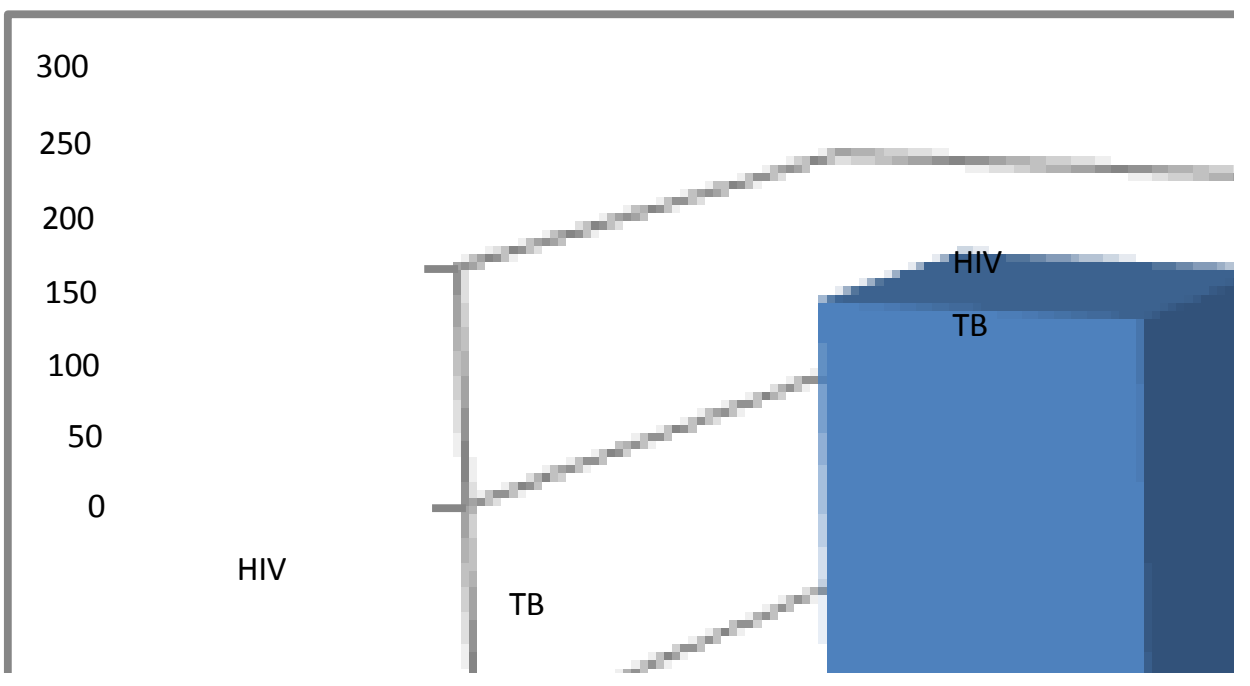


Fig.2 Rifampicin sensitive and rifampicin resistance in CBNAAT positive cases

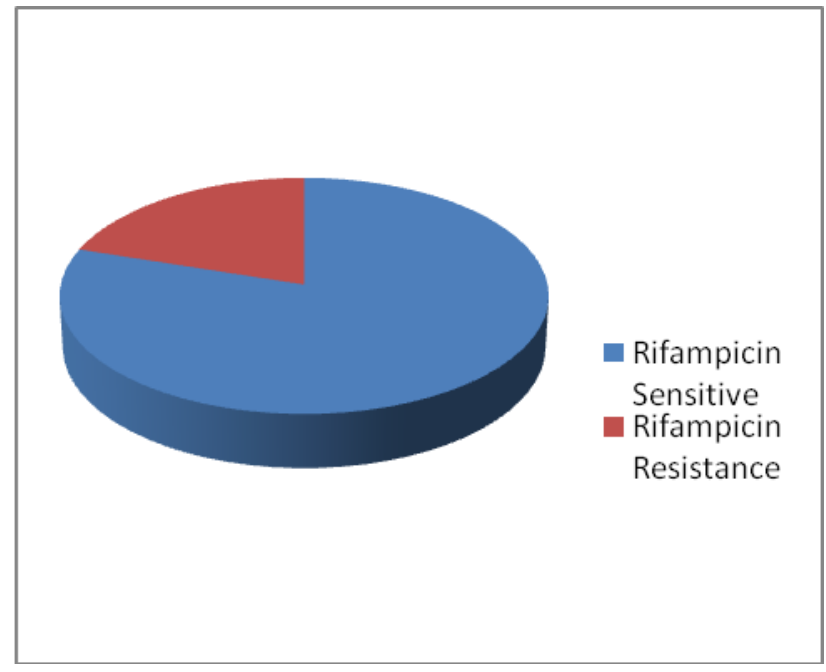

People living with HIV need early diagnosis and treatment of active TB disease. WHO recommends CBNAAT for diagnosis of pulmonary $\mathrm{TB}$ and detection of rifampicin resistance, especially in PLHIV and retreatment cases who are at risk of MDR-TB 10,11 . GeneXpert was introduced in India in 2012 by WHO whose major advantage is, it is mycobacterium tuberculosis specific, no cross reaction with non-tubercular mycobacterium occurs. Another important aspect is simultaneous detection of rifampicin resistance in 2 hours which helps in rapid initiation of MDR treatment. In our study, the prevalence of TB was found to be $39.93 \%$ which is co-relating with the findings of Neethi et al., ${ }^{12}$.

Whereas Alfred Muremo et al., ${ }^{13}$ reported a higher prevalence which is not analogous with our findings. This change in prevalence could be attributed to various factors like differences in screening of $\mathrm{TB}$, gender, educational status, occupational status, CD4 count level, WHO clinical staging ${ }^{12}$. In our study, CBNAAT detected rifampicin resistance in $20 \%$ cases. Study by Rao et al., ${ }^{6}$ and Sethi et al., ${ }^{11}$ showed rifampicin resistance in $13.55 \%$ and $27.3 \%$ cases respectively.
On the other hand, sputum microscopy for AFB being simple and economical has some disadvantages. It is highly subjective (operator dependent), sensitivity ranges from $20 \%$ to $60 \%$. This sensitivity is further reduced in PLHIV due to lower rates of caseous necrosis and sputum production ${ }^{13}$. In the present study, out of 147 HIV-TB cases, $32(11.11 \%)$ were positive by sputum microscopy for acid fast bacilli and 115 cases were reported as sputum smear negative. The consequences of this can be delayed or misdiagnosed cases, contributing to delayed treatment, increased morbidity and mortality rates and continued spread of $\mathrm{TB}$ to contacts 10 .

The appearance of many opportunistic infections correlates with the CD4 cell count as it is an important indicator of CMI. HIV infection causes a rapid decline in immune responses resulting in multiplication of the mycobacterium with in the granuloma. It is also theorized that there is an increased replication of HIV at the sites of mycobacterium infection by multiplying within the activated CD4+T cells. Hence HIV infected individuals with lower CD4 counts are more susceptible for attaining TB rather than individuals with higher CD4 counts ${ }^{14}$. 
The present study shows that the patients with CD4 count less than 200 cells/ $\mu 1$ are at higher risk of acquiring pulmonary TB. Similar correlation was reported by other studies like Kavya et al., ${ }^{15}$ and Vajpayee et al., ${ }^{16}$.

Thus the results of the present study emphasizes that co-infection with $\mathrm{TB}$ has been a major concern in HIV/AIDS patients. $\mathrm{TB}$ is the most common opportunistic infection in HIV positive patients even at higher CD4 counts and most of the time remains smear negative. Hence early diagnosis and optimum management is necessary to reduce mortality and morbidity. Utilizing culture sensitivity tests and geneXpert tests should be considered in individuals with potential risk factors.

\section{References}

1. Alfred JM, Benson RK, Stephen EM, et al., High prevalence of tuberculosis among adults with fever admitted at a tertiary hospital in north western Tanzania. Tanzania $J$ of Health Res, 2012; 14(3): 1-9.

2. Andrzej P, Marianne J, Markus S, Martin ER, Gunilla KI. PLoS pathogens. 2012; 8(2): 1-7.

3. Cain KP, McCathy KD, Heilig CM, et al., An algorithm for tuberculosis screening and diagnosis in people with HIV.N Engl J Med 2010; 362(8): 707716.

4. Chandra N, TS Durga, Devulapalli M, et al., A study on patients with TB and HIV co-infection in relation to mean CD4 counts. Ind $J$ of Pharmacy Prac, 2017; 10(2): 111-114.

5. Database for global tuberculosis report. Geneva, World Health Organization 2017.

6. Dewan R, Anuradha S, Khanna A, Garg S, Singla S, Ish P, Agarwal S; Role of cartridge-based nucleic acid amplification test (CBNAAT) for early diagnosis of pulmonary tuberculosis in HIV, JIACM, 2015; 16(2):114-7.

7. Kavya S, Anuradha K, Venkatesha D, et al., CD4 count evaluation in HIV-TB co-infection before and after antitubercular treatment. Int $J$ of Res in Med Sciences, 2017; 2(3): 1031-4.

8. Nara K, Burachat S, Waraya A, et al., HIV associated extra pulmonary tuberculosis in Thailand: epidemiology and risk factors for death. Int $J$ of Infec Dis 2009; 13: 722-9.

9. Rao DP, Sowjanya KL. Role of CBNAAT in rapid detection of mycobacterium tuberculosis in PLHIV in a highly prevalent state. $J$ Evid Based Med Healthc. 2016; 3(38).

10. Sethi S, Mewara A, Dhatwalia SK, Singh H, Yadav R, Singh K, et al., Prevalence of multidrug resistance in Mycobacterium tuberculosis isolates from HIV seropositive and seronegative patients with pulmonary tuberculosis in north India. BMC Infectious Diseases, 2013; 13:137.

11. Shankar EM, Vignesh R, Barathan M, et al., HIV-Mycobacterium tuberculosis co-infection: 'a danger couple model' of disease pathogenesis. Pathogens and disease. 2014; 70(2): 110-8.

12. Sharma SK, Soneja M, Prasad KT, et al., Clinical profile \& predictors of poor outcome of adult HIV tuberculosis patients in a tertiary care centre in north India. Indian J Med Res 2014; 139(1): 154-160.

13. Siddeswari R, Amaravathi KS, Rao NS, et al., HIV/AIDS-tuberculosis (pulmonary and extrapulmonary) coinfection: CD4 correlation. Int J of Res in Med Sciences. 2016; 4(4): 1035-9.

14. Swaminathan S, Ramachandran R, Bhaskar R, et al., Development of tuberculosis in HIV infected individuals 
in India. Int J TubercLung Dis. 2000; 4: 839-44.

15. Vajpayee M, Kanswal S, Seth $\mathrm{P}$, et al., Spectrum of opportunistic infections and profile of CD4 counts among AIDS patients in North India. Infection 2003; 31(5): 336-40.
16. WHO. Policy statement: automated real time nucleic acid amplification technology for rapid and simultaneous detection of tuberculosis and rifampicin resistance: Xpert MTB/RIF system 2011: pgs 28.

\section{How to cite this article:}

Mousumi Kilikdar, Dilip Gedam, Ashwini Pisey and Rajesh P. Karyakarte. 2019. Rapid Detection of Mycobacterium tuberculosis in PLHIV and Its Correlation with CD4 Count. Int.J.Curr.Microbiol.App.Sci. 8(07): 344-349. doi: https://doi.org/10.20546/ijcmas.2019.807.042 